\title{
THE TRIANGULAR DECOUPLING PROBLEM FOR NONLINEAR CONTROL SYSTEMS
}

\section{HENK NiJMEIJER}

Twente University of Technology, Department of Applied Mathematics, P.O. Box 217, 7500 AE Enschede, The Netherlands

(Received 3 May 1983; received for publication 20 June 1983)

Key words and phrases: Nonlinear control systems, differential geometric methods, controllability distributions, triangular decoupling.

\section{INTRODUCTION}

CONSIDER a control system of the form

$$
\begin{aligned}
& \dot{x}=A(x)+\sum_{i=1}^{m} B_{i}(x) u_{i} \\
& z_{i}=H_{i}(x), i=1, \ldots, p
\end{aligned}
$$

where $x$ are local coordinates of a smooth $n$-dimensional manifold $M, A, B_{1}, \ldots, B_{m}$ are smooth vector fields on $M$ and $H_{i}: M \rightarrow N_{i}$ is a smooth output map from $M$ to a smooth $p_{i^{-}}$ dimensional manifold $N_{i}$ for $i=1, \ldots, p$. We assume that each $H_{i}$ is a surjective submersion.

In this note we will study the (static state feedback) Triangular Decoupling Problem (T.D.P.). That is, we seek a control law of the form

$$
u=\alpha(x)+\beta(x) v
$$

where $\alpha: M \rightarrow \mathbb{R}^{m}, \beta: M \rightarrow \mathbb{R}^{m \times m}$ are smooth maps, $\beta(x)=\left(\beta_{i j}(x)\right)$ is nonsingular for all $x$ in $M$ and $v=\left(v_{1}, \ldots, \quad v_{m}\right)^{t} \in \mathbb{R}^{m}$. Let $\dot{A}(x)=A(x)+\sum_{i=1}^{m} B_{i}(x) \alpha_{i}(x)$ and $\bar{B}_{i}(x)=$ $\sum_{j=1}^{m} B_{i}(x) \beta_{j i}(x)$. Then the modified dynamics $\dot{x}=\tilde{A}(x)+\sum_{i=1}^{m} \dot{B}_{i}(x) v_{i}$ should control the output $z_{i}, i=1, \ldots, p$ sequentially, i.e. $\bar{v}_{1}$ controls $z_{1}$, possibly changing the values $z_{2}, \ldots, z_{p}$, then $\bar{o}_{2}$ controls $z_{2}$, possibly changing the values of $z_{3}, \ldots, z_{p}$, with the requirement that $z$ : be left unaffected and so forth, with $\dot{v}_{p}$ controlling $z_{p}$ without influencing $z_{1}, \ldots, z_{p-1}$ (here the $\bar{v}_{i}$ are vectors such that $\left.v_{1}, \ldots, v_{m}\right)=\left(\bar{v}_{1}, \ldots, \bar{v}_{p}\right)$ ). For linear systems the Triangular Decoupling Problem has been solved completely, see $[3,11,12,21]$. In the solution we present here we use as key tools the so called regular controllability distributions, introduced in [14]. In this way our approach completely fits in the systematic work on the generalization of the geometric approach to linear systems, see e.g. $[6-10,13-18]$. Note that in the T.D.P. the partial decoupling of the outputs is weaker than achieving complete dynamic interacting, which for a special case-the Restricted Decoupling Problem-has been solved in [16].

\section{PROBLEM FORMULATION}

Recall the following definitions, see $[6-10,14]$. 
Definition 2.1. An involutive distribution $D$ on $M$ is controlled invariant for the system (1.1a) if there exists a feedback of the form (1.2) such that the modified dynamics $\dot{x}=$ $\bar{A}(x)+\sum_{i=1}^{m} \dot{B}_{i}(x) v_{i}$ leaves $D$ invariant, i.e.

$$
\begin{aligned}
& {[\bar{A}, D] \subset D} \\
& {\left[\bar{B}_{i}, D\right] \subset D, i=1, \ldots, m .}
\end{aligned}
$$

Definition 2.2. An involutive distribution on $M$ is a regular conirollability distribution of the system (1.1a) if it is controlled invariant for the system and moreover $D=$ involutive closure of $\left\{a d_{i}^{k} \bar{B}_{i} \mid k \in \mathbb{N}, i \in l\right\}$ for a certain subset $I \subset\{1, \ldots, m\}$.

Instead of the above notion of controlled invariance we will use a slightly weaker concept, which is much easier to handle.

Definition 2.3. An involutive distribution $D$ on $M$ is locally controlled invariant for the system (1.1a) if locally around each point $x_{0} \in M$ there exists a feedback of the form (1.2) such that the modified dynamics $\dot{x}=\bar{A}(x)+\sum_{i=1}^{m} \bar{B}_{i}(x) v_{i}$ leaves $D$ invariant.

A locally controlled invariant distribution of fixed dimension can easily be characterized, see $[8,13]$.

THEOREM 2.4. Let $D$ be an involutive distribution of fixed dimension on $M$ and suppose that the distribution $D \cap \operatorname{Span}\left\{B_{1}, \ldots, B_{m}\right\}$ has fixed dimension. Then $D$ is locally controlled invariant if and only if

$$
\begin{aligned}
& {[A, D] \subset D+\operatorname{Span}\left\{B_{1}, \ldots, B_{m}\right\}} \\
& {\left[B_{i}, D\right] \subset D+\operatorname{Span}\left\{B_{1}, \ldots, B_{m}\right\}, i=1, \ldots, m}
\end{aligned}
$$

Remark 1. In theorem 2.4 the assumption that $D$ has fixed dimension is essential. Therefore one usually requires this already in definition 2.3 , see e.g. $[8,13]$. Similarly one defines a local version of definition 2.2: the regular local controllability distributions.

Finally we need a definition of output controllability, see also [16]. Consider the system (1.1a) together with an output function $H: M \rightarrow N$. Assume that $H$ is a surjective submersion. Let $D$ be the controllability distribution of $(1.1 \mathrm{a})$, see $[14,20]$, i.e. $D=$ involutive closure of $\left\{\operatorname{ad}_{A}^{k} B_{i} \mid k \in \mathbb{N}, i=1, \ldots, m\right\}$. Then we have

Definition 2.5. The system (1.1a) with output function $H: M \rightarrow N$ is output controllable if $H \cdot(D)=T N$, where $D$ is the controllability distribution of (1.1a).

Remark 2. This notion of output controllability is similar to the notion of strong accessibility for a system, [20]. Namely if we denote by $R_{t}\left(x_{0}\right)$ the reachable set of (1.1a) at time $t$ from $x_{0}$, then the system is output controllable if $H\left(R_{t}\left(x_{0}\right)\right)$ has nonempty interior in $N$.

It is now easy to see that the local version of the Triangular Decoupling Problem can be formalized, as for linear systems, in the following way: given the system $(1.1 \mathrm{a}$, b) find (if possible) a local feedback law of the form (1.2) and regular local controllability distributions 
$R_{1} \ldots, R_{p}$ such that we have

and

$$
R_{i} \subset \bigcap_{i=1}^{i-1} \operatorname{Ker} H_{j}: i=1, \ldots, p
$$

$$
R_{i}+\operatorname{Ker} H_{i^{*}}=T_{i} M
$$

In (2.1) the vacuous condition at $i=1$ just says $R_{\mathrm{l}} \subset T M$. Define $R_{i}^{*}=$ supremal regular local controllability distribution in $\cap_{j=1}^{j=1} \operatorname{Ker} H_{i^{*}}$.

Remark 3. $R_{i}^{*}$ is well defined, see $[10,14]$ and may be computed via the Controllability Subdistribution Algorithm of [10], but the dimension of $R_{i}^{*}(x)$ may change if $x$ varies in $M$.

\section{MAIN THEOREN}

THEOREM 3.1. Under the assumption that for each $i=1 \ldots \ldots, R_{i}^{*}$ as well as $R_{i}^{*} \cap \operatorname{Span}\left\{B_{1}\right.$. $\left.\ldots, B_{m}\right\}$ have fixed dimension, T.D.P. is solvable in a local fashion if and only if

$$
R_{i}^{*}+\operatorname{Ker} H_{i^{*}}=T M, i=1 \ldots, p .
$$

Proof. The necessity of (2.2) follows from the maximality of the $R_{i}^{*}$. For sufficiency we have to show that the $R_{i}^{*}$ are compatible; although each $R_{i}^{*}$ is locally controlled invariant. it by no means follows that there exists a local feedback law (1.2) which leaves each of them invariant. From $(2.1)$ it is clear that

$$
R_{i}^{*} \supset R_{2}^{*} \supset \ldots \supset R_{p}^{*}
$$

According to [19] we can choose local coordinates $\left(x_{1}, \ldots, x_{p-1}\right)$ on $M$ such that

$$
\begin{aligned}
& R_{p}^{*}=\operatorname{Span}\left\{\frac{\partial}{\partial x_{1}}\right\}, R_{p-1}^{*}=\operatorname{Span}\left\{\frac{\partial}{\partial x_{1}}, \frac{\partial}{\partial x_{2}}\right\}, \ldots, \\
& R_{1}^{*}=\operatorname{Span}\left\{\frac{\partial}{\partial x_{1}}, \ldots, \frac{\partial}{\partial x_{p}}\right\}, \text { each } x_{i} \text { possibly being a vector }
\end{aligned}
$$

$R_{p}^{*}$ is locally controlled invariant, so

$$
\left.\begin{array}{l}
{\left[A, R_{p}^{*}\right] \subset R_{p}^{*}+\operatorname{Span}\left\{B_{1}, \ldots, B_{m}\right\}} \\
{\left[B_{i}, R_{p}^{*}\right] \subset R_{p}^{*}+\operatorname{Span}\left\{B_{1}, \ldots, B_{m}\right\}, i=1, \ldots, m .}
\end{array}\right\}
$$

By theorem 2.4 this is equivalent to the fact that there exists a local feedback $u=\alpha(x)-\beta(x) v$, such that

$$
\left.\begin{array}{l}
{\left[\bar{A}, R_{p}^{*}\right] \subset R_{p}^{*}} \\
{\left[\tilde{B}_{i}, R_{p}^{*}\right] \subset R_{p}^{*}, i=1, \ldots, m}
\end{array}\right\}
$$

(here $\bar{A}$ and $\dot{B}_{i}$ are defined as in Section 1). In our local coordinates this means that

$$
\tilde{A}(x)=\left[\begin{array}{l}
\dot{A}^{1}\left(x_{1}, \ldots, x_{p+1}\right) \\
\tilde{A}^{2}\left(x_{2}, \ldots, x_{p-1}\right)
\end{array}\right], \tilde{B}_{i}(x)=\left[\begin{array}{l}
\tilde{B}_{i}^{1}\left(x_{1}, \ldots, x_{p-1}\right) \\
\bar{B}_{i}^{2}\left(x_{2}, \ldots, x_{p+1}\right)
\end{array}\right],
$$

$i=1, \ldots, m$, where $\dot{A}^{1}$, respectively $\dot{B}_{i}^{1}$, represents the first $x_{1}$-dimensional (=dim $R_{p}^{*}$ ) component of the vector field $\bar{A}$, respectively $\tilde{B}_{i}$ and $\bar{A}^{2}$, respectively $\bar{B}_{i}^{2}$, the remaining 
components of $\bar{A}$ respectively $\dot{B}_{i}$. Also $R_{p-1}^{*}$ is locally controlled invariant, so

$$
\left.\begin{array}{l}
{\left[\bar{A}, R_{p-1}^{*}\right] \subset R_{p-1}^{*}-\operatorname{Span}\left\{\bar{B}_{1}, \ldots, \bar{B}_{m}\right\}} \\
{\left[\bar{B}_{i}, R_{p-1}^{\cdot}\right] \subset R_{p-1}^{\cdot}-\operatorname{Span}\left\{\bar{B}_{1}, \ldots, \bar{B}_{m}\right\}, i=1, \ldots, m .}
\end{array}\right\}
$$

By using the second component of the vector fields $\bar{A}$ and $\bar{B}_{i}$ as in (3.5) and the fact that the dimension of $R_{p-1}^{*} \cap \operatorname{Span}\left\{\bar{B}_{1}, \ldots, \bar{B}_{m}\right\}$ modulo $R_{p}^{*}$ equals the dimension of $R_{p}^{*}-1 \cap \operatorname{Span}\left\{\bar{B}_{1}\right.$, $\left.\ldots, \tilde{B}_{m}\right\}$ minus the dimension of $R_{p}^{*} \cap \operatorname{Span}\left\{\tilde{B}_{1}, \ldots, \bar{B}_{m}\right\}$, i.e. is constant by assumption. we deduce, according to $[6,8,13]$, that we can find a local feedback $v=\bar{\alpha}(x)+\dot{\beta}(x) \bar{v}$ such that the new vector fields $\bar{A}$ and $\bar{B}_{i}$ satisfy (3.4) as well as

$$
\left.\begin{array}{l}
{\left[\bar{A}, R_{p-1}^{*}\right] \subset R_{p-1}^{*}} \\
{\left[\vec{B}_{i}, R_{p-1}^{*}\right] \subset R_{p-1}^{*}, i=1, \ldots, m .}
\end{array}\right\}
$$

Or, in our local coordinates

$$
\bar{A}(z)=\left[\begin{array}{c}
\bar{A}^{1}\left(x_{1}, \ldots, x_{p+1}\right) \\
\bar{A}^{2}\left(x_{2}, \ldots, x_{p+1}\right) \\
\bar{A}^{3}\left(x_{3}, \ldots, x_{p+1}\right)
\end{array}\right], \bar{B}_{i}(x)=\left[\begin{array}{c}
\bar{B}_{i}^{1}\left(x_{1}, \ldots, x_{p+1}\right) \\
\bar{B}_{i}^{2}\left(x_{2}, \ldots, x_{p+1}\right) \\
\bar{B}_{i}^{3}\left(x_{3}, \ldots, x_{p+1}\right)
\end{array}\right],
$$

$i=1, \ldots, m$, where $\bar{A}^{1}\left(\bar{B}_{i}^{\mathrm{l}}\right)$ is the first $x_{1}$-dimensional $\left(=\operatorname{dim} R_{p}^{*}\right)$ component of $\bar{A}\left(\bar{B}_{i}\right), \bar{A}^{2}$ $\left(\bar{B}_{i}^{2}\right)$ is the second $x_{2}$-dimensional $\left(=\operatorname{dim} R_{p-1}^{*}-\operatorname{dim} R_{p}^{*}\right)$ component of $\bar{A}\left(B_{i}\right), \bar{A}^{3}\left(\bar{B}_{i}^{3}\right)$ represents the remaining component of $\bar{A}\left(\bar{B}_{i}\right)$. Notice that this second local feedback law $v=$ $\bar{\alpha}(x)+\bar{\beta}(x) \bar{v}$ is independent of $x_{1}$, i.e. $v=\bar{\alpha}\left(x_{2}, \ldots, x_{p-1}\right)+\beta\left(x_{2}, \ldots, x_{p-1}\right) \bar{v}$. Repetition of the above argument yields

$$
\bar{A}(x)=\left[\begin{array}{c}
\bar{A}^{1}\left(x_{1}, \ldots, x_{p+1}\right) \\
\bar{A}^{2}\left(x_{2}, \ldots, x_{p+1}\right) \\
\vdots \\
\bar{A}^{p}\left(x_{p}, x_{p+1}\right) \\
\bar{A}^{p+1}\left(x_{p+1}\right)
\end{array}\right], \bar{B}_{i}(x)=\left[\begin{array}{c}
\bar{B}_{i}^{1}\left(x_{1}, \ldots, x_{p+1}\right) \\
\bar{B}_{i}^{2}\left(x_{2} \ldots \ldots x_{p+1}\right) \\
\vdots \\
\bar{B}_{l}^{p}\left(x_{p}, x_{p-1}\right) \\
\bar{B}_{l}^{p-1}\left(x_{p-1}\right)
\end{array}\right]
$$

$i=1, \ldots, m$, where $\bar{A}^{j}\left(\bar{B}_{i}^{i}\right)$ represents the $j$ th $x_{j}$-dimensional component of $\bar{A}\left(B_{i}\right)$. That is. we have shown that the distributions $R_{i}^{*}$ are compatible. Next we will use the fact that the $R_{i}^{*}$ 's are regular local controllability distributions. Using this we see that (eventually after a permutation on the new input functions $\left.\left(\bar{v}_{1}, \ldots, \bar{v}_{m}\right)\right)$ there exists a partitioning of the set $\{1, \ldots, m\}$ into $p$ subsets $I_{k}, k=1, \ldots, p$ such that $I_{1}=\left\{1, \ldots, m_{i}\right\}, I_{2}=\left\{1 \ldots \ldots m_{1}\right.$. $\left.\ldots, m_{2}\right\}, \ldots, I_{p}=\{1, \ldots, m\}$ with the property $j \in I_{k} \Leftrightarrow R_{p-k-1}^{*}$ for $k=1 \ldots, \ldots$. Therefore our system after applying feedback has the form

$$
\left[\begin{array}{c}
\dot{x}_{1} \\
\dot{x} \\
\vdots \\
\dot{x}_{p} \\
\dot{x}_{p-1}
\end{array}\right]=\left[\begin{array}{c}
\bar{A}^{1}\left(x_{1}, \ldots, x_{p+1}\right) \\
\bar{A}^{2}\left(x_{2}, \ldots, x_{p+1}\right) \\
\vdots \\
\bar{A}^{p}\left(x_{p}, x_{p+1}\right) \\
\bar{A}^{p-1}\left(x_{p-1}\right)
\end{array}\right]+\sum_{i=I_{1}}\left[\begin{array}{c}
\bar{B}_{j}^{1}\left(x_{1}, \ldots, x_{p+1}\right) \\
\bar{B}_{j}^{2}\left(x_{2}, \ldots, x_{p-1}\right) \\
\vdots \\
\bar{B}_{i}^{p}\left(x_{p}, x_{p-1}\right) \\
0
\end{array}\right] \bar{v}_{j}
$$




$$
+\sum_{j \in I_{2} M_{1}}\left[\begin{array}{c}
\bar{B}_{l}^{1}\left(x_{1}, \ldots, x_{p-1}\right) \\
\dot{B}_{j}^{2}\left(x_{2}, \ldots, x_{p-1}\right) \\
0 \\
\dot{B}_{j}^{p-1}\left(x_{p-1}, x_{p}, x_{p-1}\right) \\
0 \\
0
\end{array}\right] \bar{v}_{i}+\ldots+\sum_{i \in I_{p} ! I_{p-1}}\left[\begin{array}{c}
\bar{B}_{j}^{1}\left(x_{1} \ldots, x_{p-1}\right) \\
0 \\
\vdots \\
\vdots \\
0 \\
0
\end{array}\right] \tilde{v}_{i}
$$

Furthermore we obtain from $R_{i}^{*} \subset \cap \cap_{j=1}^{i=1} \operatorname{Ker} H_{j^{*}}$ for the output functions the following partitioning

$$
\begin{aligned}
z_{1}= & H_{1}\left(x_{p}, x_{p-1}\right) \\
z_{2}= & H_{2}\left(x_{p-1}, x_{p}, x_{p-1}\right) \\
& \vdots \\
z_{p-1}= & H_{p-1}\left(x_{2}, \ldots, x_{p-1}\right) \\
z_{p}= & H_{p}\left(x_{1}, \ldots, x_{p-1}\right) .
\end{aligned}
$$

Finally we note that the condition (3.1), $R_{i}^{*}+\operatorname{Ker} H_{i^{*}}=T M$, automatically leads to the notion of output controllability. For example the matrix $\left(\partial H_{1} / \partial x_{p}\left(x_{p}, x_{p-1}\right)\right)$ has full rank and so forth.

Remarks. (i) The system (1.1a) is strongly accessible. see [20], if $R_{i}^{*}$, the supremal controllability distribution. equals $T M$. If $R_{1}^{*}=T M$ we can skip the $x_{p-1}$ component in (3.10) and (3.11). (ii) The decomposition given here is different from the cascade decomposition given in [19] (see also [9]). (iii) In some cases one can derive conditions for invertibility for the "subsystems" with $\bar{v}_{p-j}$ as input function and $z_{j}$ as output function; see [15] for a geometric interpretation of invertibility.

\section{AN EXAMPLE: THE RIGID BODY}

We will illustrate the Triangular Decoupling Problem by a simple example of controlling the rigid body. For a mathematical description of a control system on the rigid body together with various results on controllability of the system we refer to $[1,2.4 .5]$. The setting used here is similar as in [18]. Consider the system on $S O(3) \times \mathbb{R}^{3}$

$$
\begin{gathered}
\dot{R}=S(\omega) R \\
{\left[\begin{array}{l}
a_{1} \dot{\omega}_{1} \\
a_{2} \dot{\omega}_{2} \\
a_{3} \dot{\omega}_{3}
\end{array}\right]=\left[\begin{array}{ccc}
0 & \omega_{3} & -\omega_{2} \\
-\omega_{3} & 0 & \omega_{1} \\
\omega_{2} & -\omega_{1} & 0
\end{array}\right]\left[\begin{array}{l}
a_{1} \omega_{1} \\
a_{2} \omega_{2} \\
a_{3} \omega_{3}
\end{array}\right]+\left[\begin{array}{l}
1 \\
0 \\
0
\end{array}\right] u_{1}+\left[\begin{array}{l}
0 \\
1 \\
0
\end{array}\right] u_{2}+\left[\begin{array}{l}
0 \\
0 \\
1
\end{array}\right] u_{3}}
\end{gathered}
$$

where $R \in S O$ (3) represents the position of a rigid body with respect to an inertial set of axes in $\mathbb{R}^{3} . \omega=\left(\omega_{1}, \omega_{2}, \omega_{3}\right)^{t} \in \mathbb{R}^{3}$ is the angular velocity of the rigid body, $\left(u_{1}, u_{2}, u_{3}\right)^{t}$ are the 
controls of the system and

$$
S(\omega)=\left[\begin{array}{ccc}
0 & \omega_{3} & -\omega_{2} \\
-\omega_{3} & 0 & \omega_{1} \\
\omega_{2} & -\omega_{1} & 0
\end{array}\right] .
$$

As output functions we consider

$$
\left.\begin{array}{l}
z_{1}=H_{1}(t, \omega)=\text { last row of the matrix } R \\
z_{2}=H_{2}(r, \omega)=\text { second row of } R,
\end{array}\right\}
$$

i.e. $H_{1}: S O(3) \times \mathbb{R}^{3} \rightarrow S^{2}$ and $H_{2}: S O(3) \times \mathbb{R}^{3} \rightarrow S^{2}$. Similar as in [18] we will first solve a simpler T.D.P., namely let $r=\left(r_{1}, r_{2}, r_{3}\right)^{t}$ be the first column of $R$. Then (4.1) reduce to

$$
\left[\begin{array}{c}
\dot{r}_{1} \\
\dot{r_{2}} \\
\dot{r}_{3} \\
\dot{\omega}_{1} \\
\dot{\omega}_{2} \\
\dot{\omega}_{3}
\end{array}\right]=\left[\begin{array}{c}
\omega_{3} r_{2}-\omega_{2} r_{3} \\
-\omega_{3} r_{1}+\omega_{1} r_{3} \\
\omega_{2} r_{1}-\omega_{1} r_{2} \\
b_{1} \omega_{2} \omega_{3} \\
b_{2} \omega_{1} \omega_{3} \\
b_{3} \omega_{1} \omega_{2}
\end{array}\right]+\left[\begin{array}{l}
0 \\
0 \\
0 \\
a_{1}^{-1} \\
0 \\
0
\end{array}\right] u_{1}+\left[\begin{array}{l}
0 \\
0 \\
0 \\
0 \\
a_{2}^{-1} \\
0
\end{array}\right] u_{2}+\left[\begin{array}{l}
0 \\
0 \\
0 \\
0 \\
0 \\
a_{3}^{-1}
\end{array}\right] u_{3}
$$

where $b_{1}=a_{1}^{-1}\left(a_{2}-a_{3}\right), b_{2}=a_{2}^{-1}\left(a_{3}-a_{1}\right)$ and $b_{3}=a_{3}^{-1}\left(a_{1}-a_{2}\right)$. Instead of (4.2) we obtain:

$$
\left.\begin{array}{l}
z_{1}=\bar{H}_{1}(r, \omega)=r_{3} \\
z_{2}=\bar{H}_{2}(r, \omega)=r_{2}
\end{array}\right\}
$$

According to theorem 3.1 we only have to compute the supremal regular controllability distribution $R_{2}^{*}$ in $\operatorname{Ker} H_{1^{*}}$. For this we first compute the supremal controlled invariant distribution $D$ in $\operatorname{Ker} H_{1}{ }^{*}$.

Then, see [18], $D=\operatorname{Span}\left\{X_{1}, X_{2}\right\}$ where

$$
X_{1}(r,(1))=\left[\begin{array}{l}
0 \\
0 \\
0 \\
0 \\
0 \\
1
\end{array}\right] X_{2}(r, \omega)=\left[\begin{array}{c}
r_{2} \\
-r_{1} \\
0 \\
\omega_{2} \\
-\omega_{1} \\
0
\end{array}\right] .
$$

Now it is straightforward to show that $D$ is also a regular controllability distribution and therefore we obtain $R_{2}^{*}=D$ (see also [10]). Note that the dimension of $R_{2}^{*}$ is not fixed on $S O(3) \times R^{3}$, but on the open submanifold of $S O(3) \times R^{3}$ where $r_{1} r_{2} \omega_{1} \omega_{2} \neq 0$ we certainly have that $R_{2}^{*}$ has fixed dimension and $R_{2}^{*}+\operatorname{Ker} \hat{H}_{2^{*}}=T\left(S O(3) \times \mathbb{R}^{3}\right)$. Finally we note that the system (4.3) is strongly accessible, i.e. $R_{1}^{*}=T\left(S O(3) \times \mathbb{R}^{3}\right)$, see e.g. [4, 5], and thus $R_{1}^{*}+\operatorname{Ker} \dot{H}_{i *}=T\left(S O(3) \times R^{3}\right)$. Therefore by theorem 3.1 the T.D.P. is solvable. The decou- 
pling feedback law is given by, see [18],

$$
\left[\begin{array}{l}
u_{1} \\
u_{2} \\
u_{3}
\end{array}\right]=\left[\begin{array}{c}
a_{1}\left(1-b_{1}\right) \omega_{2} \omega_{3} \\
-a_{2}\left(1+b_{2}\right) \omega_{1} \omega_{3} \\
0
\end{array}\right]+\left[\begin{array}{cc}
\omega_{2} & \omega_{1} \\
-\omega_{1} & \omega_{2} \\
0 & 0
\end{array}\right] \begin{aligned}
& 0 \\
& 0 \\
& 1
\end{aligned}\left[\begin{array}{c}
v_{1} \\
v_{2} \\
v_{3}
\end{array}\right] .
$$

Finally we see that by the same coupe de grâce as in [18] this feedback law (4.6) also solves the Triangular Decoupling Problem for the system $(4.1,2)$ on the open and dense submanifold of $S O(3) \times \mathbb{R}^{3}$ where $r_{1} r_{2} \omega_{1} \omega_{2} \neq 0$.

\section{CONCLUSION}

By generalizing the geometric approach to linear systems theory, we were able to solve the Triangular Decoupling Problem for nonlinear systems. Although it takes some more effort we think that several other "geometric" synthesis problems can be formulated and solvedin a local fashion-by the same techniques used in this paper.

\section{REFERENCES}

1. Baillieul J. \& BrocketT R. W., Controllability and observability of polynomial dynamical systems, Nonlinear Analysis 5, 543-552 (1981).

2. BAILLIEUL J. \& BROCKETT R. W., A controllability result with an application to rigid body orientation, in $21 s t$ Midwest Symposium on Circuits and Systems (Edited by H. W. H.ALE \& A. N. MiCHEL), pp. 11+117. Iowa State University E.E. Dept. (1978).

3. BASILE G. \& MARRO G., A state space approach to noninteracting controls, Ricerche di Automatica 1, 68-77 (1970)

4. Crouch P. E., Bornard B., Pritchard A. J. \& Carmichael $\therefore$.. An appraisal of nonlinear analytic systems. with applications to attitude control of a spacecraft, report to E.S.T.E.C., by Applied Systems Studies (1980).

5. CROuCH P. E. \& CARMichaEl N., Application of linear analytic systems theory to attitude control, report to E.S.T.E.C., by Applied Systems Studies (1981).

6. HiRsChORN R. M., $(A, B)$-Invariant distributions and disturbance decoupling of nonlinear systems, SIAMJ. Control Optim. 19, 1-19 (1981).

7. Isidori A., Krener A. J., Gori-Giorgi C. \& Monaco S., Vonlinear decoupling via feedback: a differential geometric approach, IEEE-Trans. Aut. Control 26, 331-345 (1981).

8. Istuori A., Krener A. J., Guki-Giorgi C. \& Monaco S., Locally ( $f . g)$-invariant distributions. Syst. Control Lett. 1, 12-15 (1981).

9. IsIDORI A., KRENER A. J., GORI-GIORGI C. \& MONACO S.. The observability of cascade connected nonlinear systems, paper presented at IFAC conference, Kyoto, Japan (1981).

10. KRENER A. J. \& ISIDORI A., (Adf,g) Invariant and controllability distributions, in Feedback Control of Linear and Nonlinear Systems, Lecture Notes in Control and Information Sciences Vol. 39, pp. 157-164 (1982).

11. Morse A. S. \& WONHAM W. M., Triangular decoupling of linear multivariable systems, IEEE-Trans. Aut. Control 15, 447-449 (1970).

12. Morse A. S. \& Wonham W. M., Status of Noninteracting Control, IEEE-Trans. Aut. Control 16, 568-581 (1971).

13. NijMeiser H., Controlled invariance for affine control systerns, Int. J. Control 34, 825-833 (1981).

14. NuMeIJer H., Controllability distributions for nonlinear control systems, Syst. Control Lett. 2, 122-129 (1982).

15. NIJMEIJER H., Invertibility of affine nonlinear control systems: a geometric approach. Syst. Control Lett. 2 , $163-168(1982)$.

16. NIJMEIJER H., Feedback decomposition of nonlinear control systems, IEEE-Trans. Aut. Control 28, 861-863 (1983).

17. NiJMeiver H. \& VAN Der SCHAFt A. H., Controlled invariance for nonlinear systems, IEEE-Trans. Aut. Control 27, 904-914 (1982).

18. Nijmetjer H. \& VAN Der Schaft A. J., Controlled invariance for nonlinear systems, two worked examples, IEEE-Trans. Aut. Control (to appear).

19. RESPONDEK W., On decomposition of nonlinear control systems. Syst. Control Lett. 1, 301-308 (1982).

20. SUSSMANN H. J. \& JURDJEVIC V., Controllability of nonlinear systems, J. diff. Eqns. 12, 95-116 (1972)

21. Woxham W. M., Linear Multivariable: a Geometric Approach, 2nd edition, Springer, Berlin (1979). 